\title{
Successful identification of pathogens by polymerase chain reaction (PCR)-based electron spray ionization time-of-flight mass spectrometry (ESI-TOF-MS) in culture-negative periprosthetic joint infection.
}

Christina L Jacovides

Rothman Institute of Orthopedics, Thomas Jefferson University Hospital

Rachael Kreft

Center for Genomic Sciences, Allegheny Singer Research Institute

Bahar Adeli

Rothman Institute of Orthopedics, Thomas Jefferson University Hospital Eollow this and additional works at: https://jdc.jefferson.edu/rothman_institute

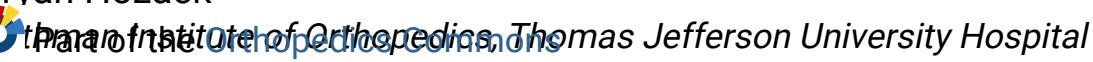

\section{at bow how access to this document benefits you}

Center for Genomic Sciences, Allegheny Singer Research institute

\section{Recommended Citation}

sacovidess Christipąitio Kreftutharshael; Adeli, Bahar; Hozack, Bryan; Ehrlich, Garth D; and Parvizi, Javad, "Successful identification of pathogens by polymerase chain reaction (PCR)-based electron spray ionization time-of-flight mass spectrometry (ESI-TOF-MS) in culture-negative periprosthetic joint infection." (2012). Rothman Institute Faculty Papers. Paper 22.

https://jdc.jefferson.edu/rothman_institute/22

This Article is brought to you for free and open access by the Jefferson Digital Commons. The Jefferson Digital Commons is a service of Thomas Jefferson University's Center for Teaching and Learning (CTL). The Commons is a showcase for Jefferson books and journals, peer-reviewed scholarly publications, unique historical collections from the University archives, and teaching tools. The Jefferson Digital Commons allows researchers and interested readers anywhere in the world to learn about and keep up to date with Jefferson scholarship. This article has been accepted for inclusion in Rothman Institute Faculty Papers by an authorized administrator of the Jefferson Digital Commons. For more information, please contact: JeffersonDigitalCommons@jefferson.edu. 
Authors

Christina L Jacovides, Rachael Kreft, Bahar Adeli, Bryan Hozack, Garth D Ehrlich, and Javad Parvizi 


\title{
Successful Identification of Pathogens by Polymerase Chain Reaction (PCR)-Based Electron Spray Ionization Time-of-Flight Mass Spectrometry (ESI-TOF-MS) in Culture-Negative Periprosthetic Joint Infection
}

\author{
Christina L. Jacovides, BS, Rachael Kreft, BS, RN, Bahar Adeli, BA, Bryan Hozack, BA, \\ Garth D. Ehrlich, PhD, and Javad Parvizi, MD, FRCS \\ Investigation performed at the Rothman Institute of Orthopedics at Thomas Jefferson University Hospital, \\ Philadelphia, and the Allegheny Singer Research Institute, Pittsburgh, Pennsylvania
}

\begin{abstract}
Background: The diagnosis of periprosthetic joint infection poses many challenges, one of which is the difficulty of isolating the infecting organism. Recently, a sophisticated modality (the Ibis Biosciences T5000 biosensor system) has been introduced that uses pan-domain primers in a series of polymerase chain reactions (PCRs) to identify and speciate essentially all bacteria and fungi as well as to identify key antibiotic resistance genes. We investigated the role of the Ibis in identifying infecting organisms in cases of known and suspected periprosthetic joint infection.
\end{abstract}

Methods: Synovial fluid specimens were collected prospectively from eighty-two patients undergoing eighty-seven arthroplasty procedures (sixty-five knee revisions, fifteen hip revisions, and seven primary knee arthroplasties) and were sent for both conventional culture and Ibis analysis. The surgeon's clinical determination of the cause for revision arthroplasty was failure due to infection in twenty-three cases and noninfectious failure in fifty-seven cases.

Results: In the twenty-three cases that were considered on clinical grounds to involve a periprosthetic joint infection, the Ibis detected the same pathogen isolated by conventional culture in seventeen of eighteen cases and also detected one or more organisms in four of the five culture-negative cases. In addition, the Ibis detected organisms in fifty (88\%) of the fiftyseven cases in which revision arthroplasty was performed for a presumed noninfectious failure.

Conclusions: The Ibis technology was not only effective at detecting organisms in cases of suspected periprosthetic joint infection in which cultures were negative, but it also suggested that many of the revision arthroplasty cases that have previously been considered to be purely aseptic may have a component of unrecognized, subclinical infection.

Level of Evidence: Diagnostic Level III. See Instructions for Authors for a complete description of levels of evidence.

$\mathrm{T}$ he diagnosis of periprosthetic joint infection is a challenge. Since no highly accurate diagnostic method exists, clinicians must rely on some combination of clinical suspicion, serology, culture, and basic molecular techniques ${ }^{1,2}$. Identification of the infecting organism is most commonly accomplished with use of culture. However, since culture cannot always provide accurate information about the existence and virulence of microorganisms in an infected joint, the diagnosis of periprosthetic joint infection may remain elusive ${ }^{1-6}$.

Inaccuracy of culture results includes false-positive and false-negative results. Obtaining multiple cultures and also incorporating laboratory findings and clinical symptoms in a

Disclosure: None of the authors received payments or services, either directly or indirectly (i.e., via his or her institution), from a third party in support of any aspect of this work. One or more of the authors, or his or her institution, has had a financial relationship, in the thirty-six months prior to submission of this work, with an entity in the biomedical arena that could be perceived to influence or have the potential to influence what is written in this work. No author has had any other relationships, or has engaged in any other activities, that could be perceived to influence or have the potential to influence what is written in this work. The complete Disclosures of Potential Conflicts of Interest submitted by authors are always provided with the online version of the article. 
The Journal of Bone \& Joint Surgery - Jbjs.org Volume 94-A · Number 24 - December 19, 2012
Identification of Pathogens by Mass Spectrometry in Culture-Negative Periprosthetic Joint Infection diagnosis of periprosthetic joint infection can help to reduce the impact of false-positive culture results on the course of treatment. False negatives present a more onerous problem ${ }^{4,6}$, particularly in patients with clear clinical signs of infection, since antibacterial treatment cannot be appropriately tailored against a specific infecting organism ${ }^{7,8}$. Moreover, justifying additional surgical procedures in the absence of positive identification of a pathogen is psychologically difficult for both the patient and the surgeon.

The use of molecular techniques to identify pathogenic species in orthopaedic patients was first employed to address the limitations of standard cultures ${ }^{9-13}$. Polymerase chain reaction (PCR)-based assays represent a rapid, sensitive means of identifying pathogens when standard culture methods may fail ${ }^{9-11,13,14}$. Historically, this technique involved amplification of DNA with use of either species-specific primers or a single pan-domain primer pair for the gene for $16 \mathrm{~S}$ ribosomal RNA (rRNA). These approaches led, respectively, to high false-negative and high false-positive rates $^{12}$. More recent efforts have improved the specificity of amplification-based methods by using messenger RNA (mRNA) as a target ${ }^{15}$. To increase the speed of diagnosis, a dry-reagent dipstick that can assess the presence of common infectious organisms within minutes has also been introduced ${ }^{16}$.

The present study, which is an extension of a previous study and includes some patients previously reported on $^{17}$, investigated the role that a novel pan-domain DNA-based amplification and analysis system, the Ibis T5000 biosensor (Isis Biosciences, Carlsbad, California [now a subsidiary of Abbott Laboratories, Abbott Park, Illinois]), may be able to play in improving the diagnosis of periprosthetic joint infection. We determined how frequently the Ibis identified the same organism and resistance genes as conventional culture in patients undergoing revision arthroplasty, as well as how frequently it identified additional organisms or resistance genes not seen by conventional culture. We then compared components of the assay results (identification of organisms, confidence level of identification, and number of genomes) with the clinical findings and assessed the sensitivity, specificity, positive and negative predictive values, and accuracy of the Ibis in the diagnosis of periprosthetic joint infection. We additionally analyzed the findings in primary total knee arthroplasty samples and in samples from consecutive surgical procedures on the same joint.

\section{Materials and Methods}

A 11 patients undergoing primary or revision knee or hip arthroplasty from A February 2009 to May 2010 were eligible for recruitment into this study, which was approved by our institutional review board. A total of 361 revisions (201 hips and 160 knees) were performed in 341 patients during this period. We prospectively collected intraoperative synovial fluid specimens from eighty-two of these patients during eighty-seven surgical procedures (sixty-five revision total knee arthroplasties in sixty-three patients, fifteen revision hip arthroplasties in fifteen patients, and seven primary total knee arthroplasties in five patients). (Two patients underwent bilateral primary total knee arthroplasty, one patient underwent bilateral revision total knee arthroplasty, one patient underwent two revision arthroplasties of the same knee, and one patient underwent a primary and a revision arthroplasty of the same knee.)

These patients had undergone routine workups for infection (in accordance with institutional protocols) that were standardized among all surgeons. Intraoperative synovial fluid and/or tissue specimens were sent for culture, and synovial fluid was sent for analysis with use of the Ibis technology. Joints were diagnosed as infected or uninfected by the treating surgeon on the basis of clinical suspicion (clinical judgment in the office and operating room) and a combination of tests involving serum (erythrocyte sedimentation rate [ESR] and C-reactive protein [CRP] level), synovial fluid (cell count with differential and culture), and tissue (culture).

Synovial fluid was collected in the operating room with use of a sterile syringe, transferred to sterile cryogenic vials on ice, and flash-frozen in liquid nitrogen at $-140^{\circ} \mathrm{C}$ within one hour of collection. Samples were stored and shipped in batches to a different institution for the Ibis analyses.

After shipping, the synovial fluid samples were stored at $-80^{\circ} \mathrm{C}$ until DNA extraction was performed. A 1-mL aliquot was centrifuged at 10,000 rpm for three minutes, $900 \mu \mathrm{L}$ of supernatant was removed, and lysis buffer (Buffer ATL; Qiagen, Valencia, California) and proteinase $\mathrm{K}$ were added to the remaining sample. The sample was incubated at $56^{\circ} \mathrm{C}$ until lysis was achieved (as determined by the absence of visible tissue structure). Nucleic acid from the lysed sample was extracted with use of a DNeasy Blood \& Tissue kit (catalog number 69506; Qiagen). A 10- $\mu \mathrm{L}$ aliquot of the extracted DNA sample was loaded into each of sixteen different primer wells on a BAC (bacteria, antibiotic resistance, Candida) detection PCR plate (catalog number 05N13-01; Abbott Molecular, Des Plaines, Illinois). The BAC detection plate is a ninety-six-well microtiter plate that analyzes six specimens at a time and provides for the speciation of all bacterial organisms and Candida species as well as the detection of several key antibiotic resistance markers (including vanA and vanB [vancomycin resistance] in enterococci, KPC [carbapenem resistance] in gramnegative bacteria, and mecA [methicillin resistance] in staphylococci). Once the PCR process was complete, the plate was loaded onto the Ibis T5000 instrument. The PCR products were desalted in a ninety-six-well plate format and were sequentially introduced with use of electron spray ionization into the time-of-flight mass spectrometer (ESI-TOF-MS). The resultant spectral signals were processed to determine the masses associated with each of the PCR products with sufficient accuracy that the base composition of each amplicon could be unambiguously deduced.

The Ibis technique is based on the principle that, despite their great genomic diversity, microbial organisms have genomes containing sets of common genes that are shared at various taxonomic levels and that can provide targets for detection and speciation. The broadest-range primers are designed to amplify a product from an entire domain of microbial life (e.g., all eubacteria), whereas more specific primers are designed to identify genera and species in major pathogenic groups, as well as genes that determine antibiotic resistance. Most samples produce a mixture of products, reflecting the diversity of the original mixture of organisms present in the starting sample.

All positive findings from the Ibis were confirmed by deep 16S rRNA gene sequencing with use of a sequencing system from 454 Life Sciences (Branford, Connecticut). We designed a unique sequence-based bar-coded $16 \mathrm{~S}$ PCR primer pair for each sample to provide for multiplexing and bioinformatic deconvolution of the results following sequencing. Primers were generated by combining the sequencing primer key (454 Life Sciences), an exclusive 10-base MID (multiplex identifier) adaptor (454 Life Sciences), and a sequence targeted to the 16S rRNA gene. DNA from each orthopaedic sample and DNA from negative controls (processed identically to the specimens) were used as the PCR templates. The PCR procedures were performed in $50 \mu \mathrm{L}$ of solution containing 1X High Fidelity PCR Buffer, $2.0 \mathrm{mM} \mathrm{MgCl}$ solution, $0.8 \mathrm{mM}$ dNTP Mix, and 1 U Platinum Taq DNA Polymerase High-Fidelity (all from Invitrogen, Carlsbad, California), $0.4 \mu \mathrm{M}$ of each primer (IDT, Coralville, Iowa), and $5 \mu \mathrm{L}$ of DNA extract. Cycling involved an initial denaturation step at $94^{\circ} \mathrm{C}$ for two minutes followed by thirty cycles of $94^{\circ} \mathrm{C}$ for thirty seconds, $55^{\circ} \mathrm{C}$ for thirty seconds, and $72^{\circ} \mathrm{C}$ for sixty seconds, followed by a final extension for seven minutes at $72^{\circ} \mathrm{C}$. Five microliters of the PCR products was visualized on a $1 \%$ agarose gel. Positive PCR products were purified with use of an Agencourt AMPure XP system (Beckman Coulter, Beverly, Massachusetts) and quantified with use of a Quant-iT PicoGreen dsDNA Assay Kit (Invitrogen). All primers that produced 16S PCR fragments were diluted to an equimolar concentration $(48 \mathrm{pg} / \mu \mathrm{L})$. The PCR products were then pooled into two groups of samples to 
The Journal of Bone \& Joint Surgery $\cdot$ JBjS. org Volume 94-A · Number 24 - December 19, 2012

Identification of Pathogens by Mass Spectrometry in Culture-Negative Periprosthetic Joint Infection

TABLE I Demographic and Laboratory Characteristics of Presumed Infected and Uninfected Patients

\begin{tabular}{|c|c|c|c|c|}
\hline Characteristic* & \multicolumn{3}{|c|}{ Revision } & Primary $\dagger$ \\
\hline \multicolumn{5}{|l|}{ Demographics } \\
\hline Age (yr) & $69 \pm 10$ & $64 \pm 11$ & 0.06 & $58 \pm 10$ \\
\hline Sex (\% male) & 39.1 & 54.4 & 0.32 & 40.0 \\
\hline $\mathrm{BMI}\left(\mathrm{kg} / \mathrm{m}^{2}\right)$ & $31 \pm 5$ & $33 \pm 7$ & 0.15 & $40 \pm 6$ \\
\hline \multicolumn{5}{|l|}{ Laboratory values } \\
\hline $\operatorname{ESR}(m m / h r)$ & $80 \pm 28$ & $26 \pm 17$ & $<0.0001$ & \\
\hline $\mathrm{CRP}(m g / d L)$ & $9.3 \pm 8.8$ & $0.9 \pm 0.8$ & $<0.0001$ & \\
\hline Synovial WBC (per $m L$ ) & $21,000 \pm 24,000$ & $1200 \pm 2600$ & $<0.0001$ & \\
\hline Synovial PMN (\%) & $84 \pm 20$ & $34 \pm 31$ & $<0.0001$ & \\
\hline Purulence $\neq$ (\% purulent) & 91 & 0 & $<0.0001$ & \\
\hline
\end{tabular}

create two duplicate amplicon libraries. Sequencing of the amplicon libraries was then performed with use of the GS FLX Titanium series (454 Life Sciences) according to the manufacturer's guidelines. Following conclusion of the $16 \mathrm{~S}$ sequencing run, reads were separated by MID (i.e., by sample), and the MIDs were stripped from the reads. The reads for each sample were then analyzed with use of an online RDP (Ribosomal Database Project) classifier tool (http:// rdp.cme.msu.edu/classifier/classifier.jsp).

For each sample tested, the Ibis results gave the organisms and resistance genes identified, the confidence level of each identification (on a scale from 0.0 to 1.0 ), and the concentration of each product (number of genomes found per well). We analyzed the degree of correspondence between each of these output parameters from the Ibis with the surgeon's diagnosis of the presence of infection.

\section{Statistical Methods}

Descriptive statistics (means and standard deviations for continuous variables, and frequency distributions for categorical variables) were calculated. The $t$ test was used to compare means for continuous variables, and chi-square analysis was used for categorical variables. A p value of 0.05 was considered significant. Sensitivity and specificity analyses were conducted, with surgeon judgment regarding the presence or absence of periprosthetic joint infection as the gold standard.

\section{Source of Funding}

No authors or affiliated institutions received financial support related to this study.

\section{Results}

$\mathrm{T}$ wenty-three of the included arthroplasties were revised for presumed failure due to infection, and fifty-seven were revised for presumed aseptic failure. Samples from seven primary total knee arthroplasties were also analyzed (Table I).

We began our analysis by examining the correspondence between the conventional culture results and Ibis results in the infected and uninfected groups (Fig. 1 and Table II). Conventional culture isolated organisms in eighteen of the twenty-three cases in which revision was due to a suspected infection; multiple genera were identified in three cases. Of note, in four of these eighteen cases the organism isolated was found in a single culture with "very light" or "broth only" growth; at our institution, such culture results raise concerns of contamination and are conventionally considered "false" positives. The Ibis detected the same organism as culture in seventeen of the eighteen cases with positive cultures, and also identified an additional organism in three of these seventeen cases. In the eighteenth case, the Ibis detected Streptococcus, Enterococcus, and Treponema species, whereas conventional culture isolated Peptostreptococcus and Corynebacterium species. Importantly, no patient for whom conventional culture isolated an organism had a negative Ibis result. The Ibis also identified the mecA gene in all eight of the patients in whom methicillin-resistant Staphylococcus was isolated by culture. In fact, one of these methicillin-resistant Staphylococcus cultures (which the Ibis confirmed) was one of the cultures that was suspected to be a false positive.

In the remaining five culture-negative cases of periprosthetic joint infection, the Ibis identified organisms in four cases. Staphylococcus and/or Streptococcus species plus multiple other genera were identified in two cases, Enterococcus was identified in one case, and Acinetobacter was identified in the remaining case. Intraoperative purulence had been noted in three of these four Ibis-only positive cases, and both the ESR and CRP values were elevated in the fourth case. The Ibis identified no organisms in the remaining culture-negative case that was considered infected at the time of surgery. This patient had undergone a single-stage exchange arthroplasty for infection four months prior to resection (at which time fluid was collected) and had elevated ESR and CRP values at the time of the resection surgery, a moderate amount of cloudy fluid in the knee but no purulence, and six negative cultures. She underwent reimplantation three 
The Journal of Bone \& Joint Surgery $\cdot$ Jbjs.org Volume 94-A · Number 24 - December 19, 2012

Identification of Pathogens by Mass Spectrometry in Culture-Negative Periprosthetic Joint Infection

Positive

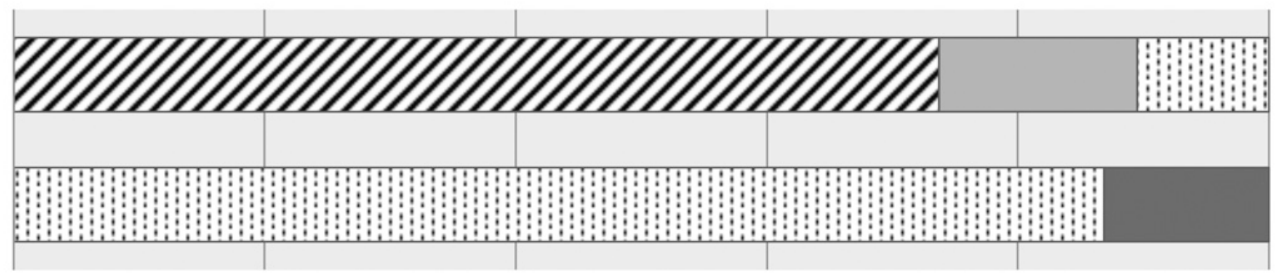

Negative

\section{Infected}

\section{Positive \\ Negative}

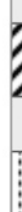
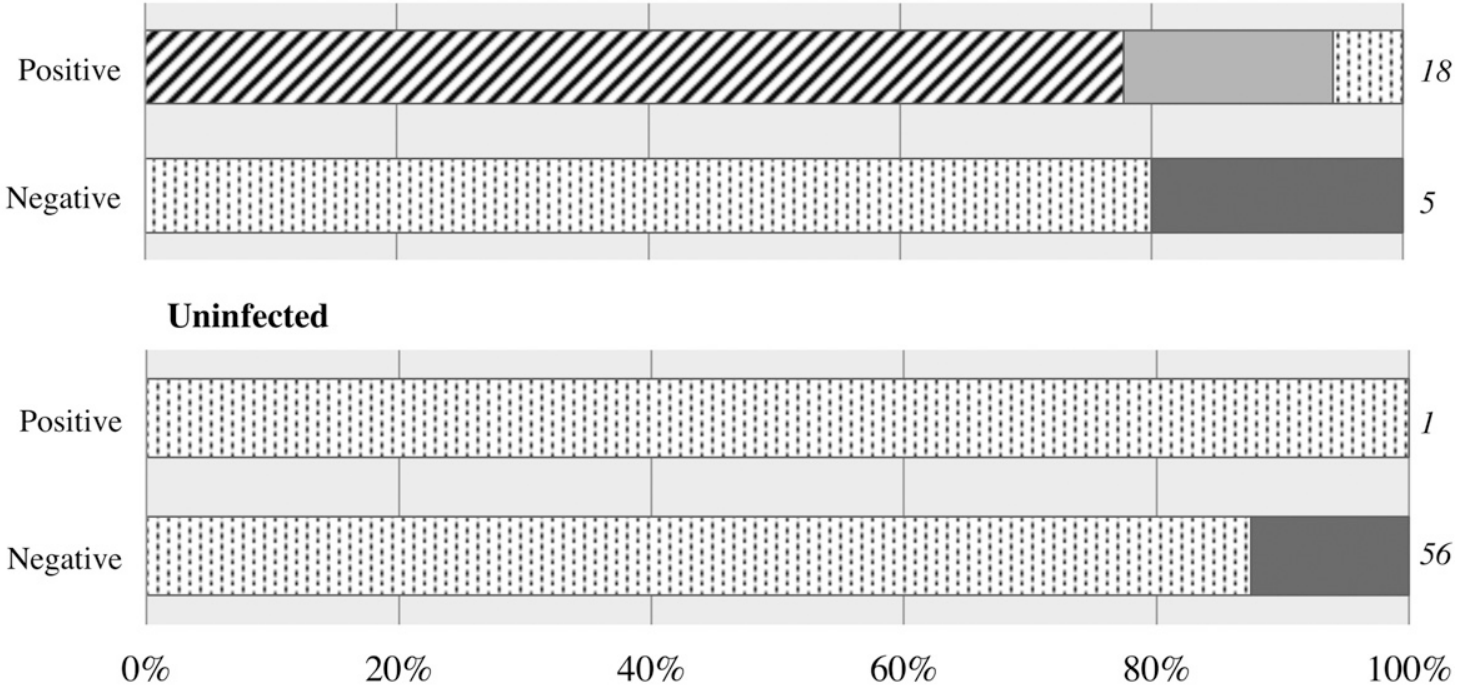

Percent of category

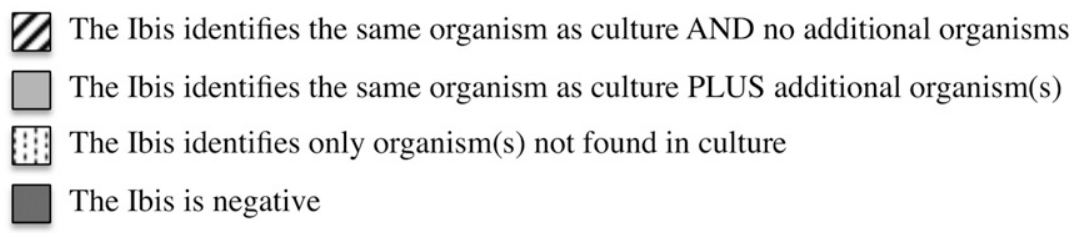

Fig. 1

Comparison of the Ibis results with results from conventional culture, shown as "Positive" or "Negative." "Positive" results include both "true" positives (substantial growth or growth on more than one plate) and "false" positives (minimal growth on a single plate). "Negative" results include both negative cultures and patients in whom no culture was taken. The number $(\mathrm{N})$ in each group is given at the far right. For each patient's sample, we determined whether the Ibis identified (1) the same organism as culture and no additional organisms, (2) the same organism as culture plus additional organism(s), (3) only organisms not found in culture, or (4) no organisms. Results are plotted as percentages within each subcategory for the total cohort as well as for the groups presumed to be infected and uninfected. Positive culture results matched well with the lbis results. Of note, four of five patients in the infected group with negative cultures had positive lbis findings. No patients in whom cultures identified an organism had negative lbis results.

months following resection, with a good outcome on long-term follow-up.

The fifty-seven cases in which revision was performed for a presumed noninfectious failure included one case in which a positive culture was believed to be a false positive, fifty-one cases with negative cultures, and five cases for which culture results could not be found. The Ibis did not identify the organism identified by the presumed false-positive culture (Propionibacterium acnes), but rather identified organisms from two other genera (Enterococcus and Campylobacter) in this patient's sample. The Ibis detected one or more organisms in forty-nine of the remaining fifty-six cases; multiple organisms were detected in twenty-four of the cases (Table II). The Ibis also identified the mecA gene and Staphylococcus species in a single case. This patient's revision arthroplasty failed six months following surgery, and she required an irrigation and debridement for subsequent periprosthetic joint infection. Examination of the medical records of the patients who underwent revision for a presumed noninfectious cause revealed that seven patients had a surgical history that involved infection and sixteen had abnormal serology and/or synovial cell counts (frankly abnormal in eight and borderline abnormal 
The Journal of Bone \& Joint Surgery $\cdot$ Jbjs.org Volume 94-A · Number 24 - December 19, 2012

Identification of Pathogens by Mass Spectrometry in Culture-Negative Periprosthetic Joint Infection

TABLE II Distribution and Comparison of Genera Identified by Culture and by the lbis

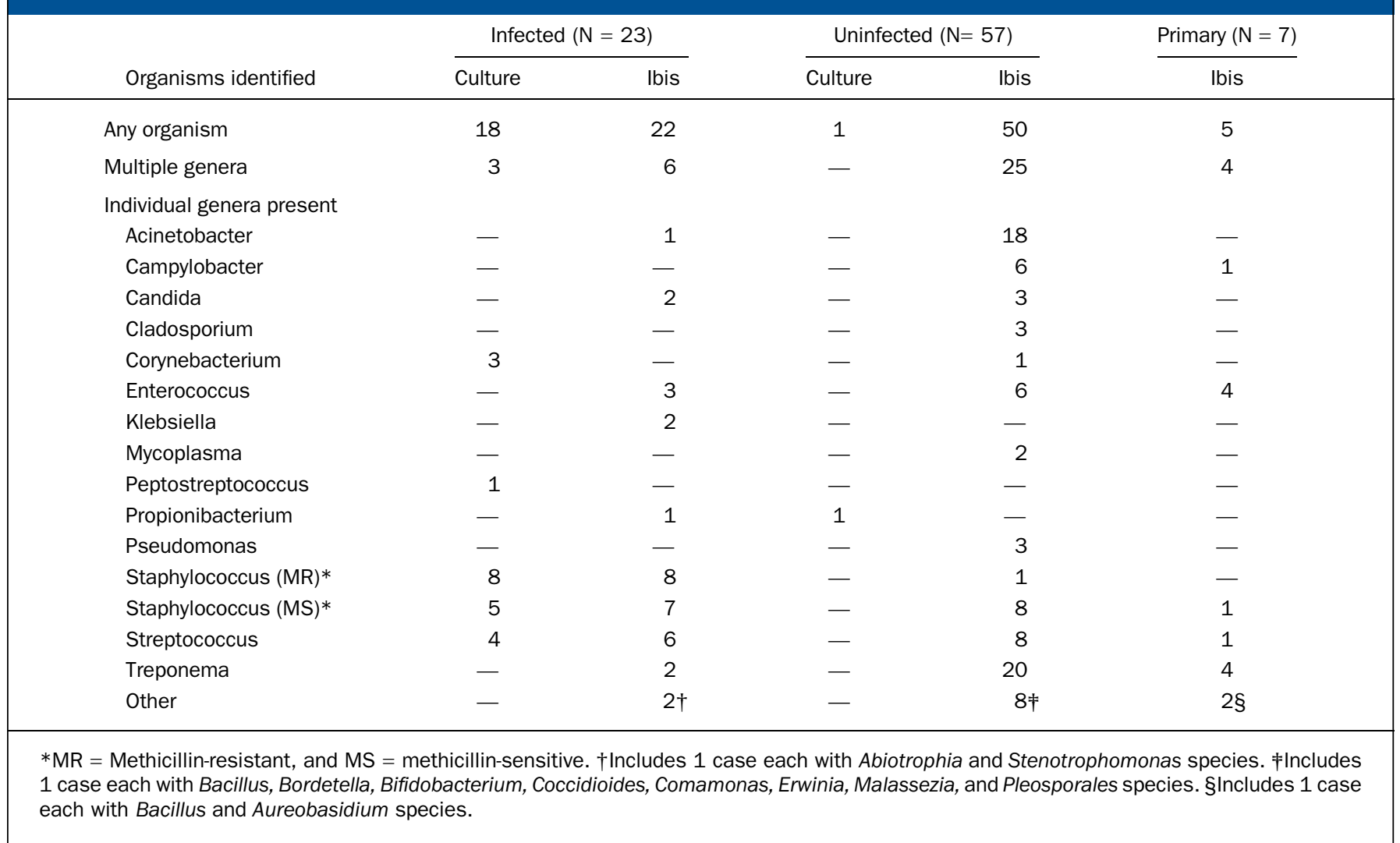

in eight). Nevertheless, the overall clinical picture had still suggested a noninfectious etiology for the arthroplasty failure in all of these patients. The only other subsequent procedures in this cohort were due to stiffness (one patient who underwent a manipulation under anesthesia) and instability (one patient whose hip was multiply revised and who ultimately required a Girdlestone procedure). The remaining patients in the uninfected group who had a positive Ibis finding have done well, without further problems or failures to date, although the maximum duration of follow-up was only 1.7 years.

We evaluated the sensitivity, specificity, positive and negative predictive value, and accuracy of each component of the Ibis results for differentiating between infected and uninfected patients (Table III). The presence of one or more organisms, the presence of staphylococci and/or streptococci, and a confidence level of $>0.7$ for the identification of any organism had the greatest sensitivity for periprosthetic joint infection $(95.7 \%, 87.0 \%$, and $95.7 \%$, respectively). The presence of mecA, a confidence level of $>0.8$ for the identification of any organism, and a maximum number of genomes per well of $>200$ had the greatest specificity for periprosthetic joint infection $(98.3 \%, 84.2 \%$, and $96.5 \%$, respectively).The presence of mecA had the greatest positive predictive value $(87.5 \%)$, and a confidence level of $>0.7$ for the identification of any organism and the presence of staphylococci and/or streptocci had the greatest negative predictive value $(96.0 \%$ and $93.3 \%$, respectively).

The Ibis results were negative in two of the seven primary total knee arthroplasty cases (five patients). Three organisms were identified in each of four cases, and one organism was identified in the remaining case (Table II). In the two patients who underwent bilateral total knee arthroplasty, the Ibis profiles differed between the two knees. In one patient, one knee had negative Ibis results and the other had Enterococcus, Treponema, and Bacillus species. In the other patient, one knee had Campylobacter species and the other had Enterococcus, Treponema, and Staphylococcus species.

In two patients, fluid samples were captured from the same knee in consecutive surgical procedures. One patient underwent primary total knee arthroplasty and then revision surgery nine months later; the other underwent two revision total knee arthroplasties involving the same knee six months apart. For the first patient, the Ibis identified Enterococcus, Treponema, and Aureobasidium species in the sample from the primary total knee arthroplasty and Streptococcus, Staphylococcus, and Acinetobacter species in the sample from the revision surgery. For the second patient, the Ibis identified Staphylococcus, Stenotrophomonas, Treponema, and Candida species at the first revision surgery and Streptococcus species at the second revision. 
The Journal of Bone \& Joint SURgery $\cdot$ JBjS.org Volume 94-A · Number $24 \cdot$ December 19,2012

Identification of Pathogens by Mass Spectrometry in Culture-Negative Periprosthetic Joint Infection

TABLE III Sensitivity, Specificity, Positive and Negative Predictive Values, and Accuracy of lbis Findings

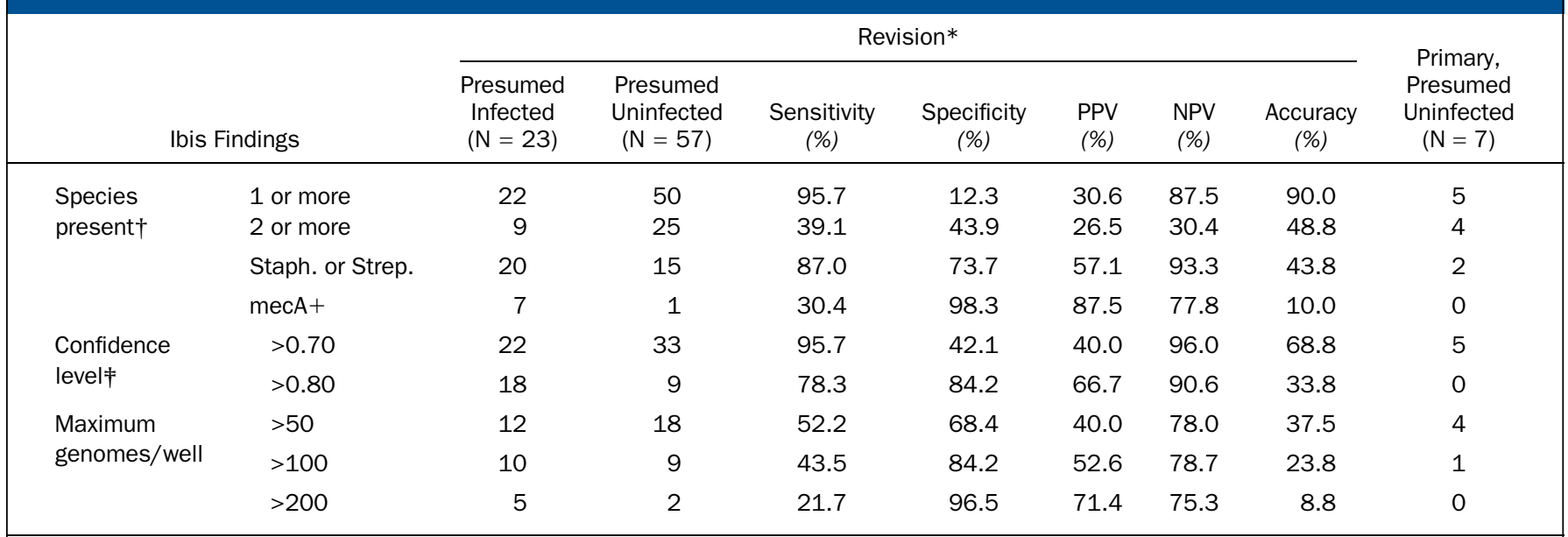

*PPV $=$ positive predictive value, and NPV = negative predictive value. †Identification of the presence of one or more organisms, two or more organisms, Staphylococcus and/or Streptococcus species, and the mecA gene sequence, respectively. ¥Highest confidence level of an identified organism.

\section{Discussion}

I $\mathrm{n}$ this study, we examined the correspondence between 1 findings from the Ibis and from conventional culture, and we also performed a preliminary assessment of the diagnostic value of the Ibis findings. We believe that the Ibis system is not only a valuable tool for the diagnosis of periprosthetic joint infection but also advances our understanding of the pathophysiology of this disease.

First, the Ibis findings agreed with the findings of the conventional cultures. No patient who had an organism identified by conventional culture had a negative Ibis result. Moreover, the Ibis identified the same organism as conventional culture in seventeen of eighteen patients in whom an infection was suspected on the basis of clinical evaluation. In the one patient in whom the specific organism identified in culture was not identified by the Ibis, the Ibis identified a different organism in the sample. Finally, the Ibis accurately identified the mecA gene in eight of eight cases of infection in which conventional culture isolated a methicillin-resistant organism. Notably, one of these cultures would likely have been considered a false positive due to minimal growth in culture.

We were very interested by the Ibis findings in patients with culture-negative infection, since one of the main goals of using molecular techniques to diagnose periprosthetic joint infection is to increase the rate of organism identification in culture-negative cases. False-negative cultures in a patient with periprosthetic joint infection may be due to a variety of factors. First, hospital culture media support only a select number of pathogens and may be unable to identify infections caused by rare or fastidious microorganisms $s^{14,18,19}$. Second, slow-growing pathogens may not be identified within the standard incubation period; moreover, increasing the incubation period would increase the rate of falsepositive results ${ }^{19,20}$. Third, formation of complex biofilms on prosthetic surfaces decreases the quantity of free-floating bac- teria, consequently leading to a greater likelihood of a negative culture $^{21-24}$. Finally, antibiotic prophylaxis can result in negative cultures ${ }^{25,26}$. The Ibis identified organisms in four of the five patients who had negative cultures at the time of revision arthroplasty but were considered by the surgeon to have an infection. This result shows promise for improving the diagnosis of patients with false-negative cultures, since it appears that the Ibis can circumvent all of these problems and make possible the identification of both the infecting organisms and resistance genes.

Despite the strong correspondence between the Ibis and culture findings in patients with culture-positive periprosthetic joint infection, we saw a very different pattern among patients who were not considered to have an infection at the time of surgery. The majority of these patients had positive Ibis findings that were subsequently confirmed by deep $16 \mathrm{~S}$ sequencing. These results suggest that our ability to detect bacteria associated with a joint is not always indicative of overt disease. In attempting to make clinical sense of these findings, we note that several studies have suggested that subclinical infections may progress very slowly, without the production of standard inflammatory markers or culturable bacteria, yet may still result in pathology that requires joint replacement ${ }^{27-30}$. Ince et al. attempted to use PCR and conventional culture to establish the presence of such "subclinical" infection in patients in whom an acetabular cup underwent revision for an apparently aseptic cause; however, an organism could be identified in only one of twenty-four cases ${ }^{31}$. In contrast, a study by Günther et al. showing that different gene products are activated in the context of infectious compared with noninfectious loosening of a joint endoprosthesi ${ }^{32}$ suggests that host factors play an important role in the pathophysiology of periprosthetic joint infection. The complete pathophysiology of arthroplasty failure in the setting of bacterial presence in the joint, however, is still under investigation. 
The Journal of Bone \& Joint Surgery · Jbjs.org Volume 94-A · Number 24 - December 19, 2012
Identification of Pathogens by Mass Spectrometry in Culture-Negative Periprosthetic Joint Infection
It remains possible that some of the patients in the ostensibly uninfected group in our cohort in fact had long-standing occult infections $s^{30}$. At least one of these patients (in whom the Ibis identified both the mecA gene and Staphylococcus species) required an irrigation and debridement for periprosthetic joint infection six months after her first revision arthroplasty. This patient also had elevated serology and synovial fluid parameters at the time of her first revision, suggesting an ongoing inflammatory process. At least four additional patients had a medical or surgical history of infection. These findings reflect the high sensitivity of the Ibis; i.e., surgeons should not take identification of an organism by the Ibis as an indication of the need for revision arthroplasty in the same way that they would if a florid infection were present. It is also clear that additional studies need to be conducted to better define the root causes of what is currently considered noninfectious loosening, since our findings suggest that occult infection could play a role in hastening this pathology.

Because of the high sensitivity of the Ibis, we questioned how individual components of the Ibis results matched against clinician-determined periprosthetic joint infection. We identified several characteristic Ibis findings that corresponded well with the presence of clinically relevant infection. We believe that better characterization of the features of a clinically relevant Ibis result will increase the immediate clinical utility of this technology. Of note, the presence of the mecA gene appeared to have the highest positive predictive value for infection, whereas both the absence of Staphylococcus or Streptococcus and a lowconfidence identification of an organism by the Ibis had the highest negative values for infection.

Finally, the findings from both the primary total knee arthroplasty patients and the patients from whom we obtained synovial fluid samples during consecutive surgical procedures were interesting. In none of the primary total knee arthroplasties did the Ibis identify the mecA gene, nor did it identify any organism at a confidence level of $>0.8$ or with $>200$ genomes per well. The presence of organisms in joints during primary arthroplasty is not surprising, given that the joint space is not sterile and may be susceptible to hematogenous seeding. Although our sample size of consecutive surgical procedures was small, it was also interesting to note the changing characteristics of the synovial flora over time in each patient's samples. Both of these results support the concept that the development of periprosthetic joint infection may involve a more complex pathophysiology than may have been previously considered.

We believe that the Ibis may play one final important role. Its use of pan-domain primers allows the placement of novel amplicons on a phylogenetic tree and comparison with existing species and their virulence patterns. This capability will not only lead to the discovery of new species and mutations within species, but it will also improve our understanding of the way in which these changes may affect diagnosis, treatment (specifically, in cases of antibiotic resistance), and patient outcomes.

There are several limitations inherent in our study. First, the duration of follow-up of many of the patients was limited, and we therefore cannot comment on associations between the findings from the Ibis and long-term outcomes. Despite this minimal follow-up, however, the Ibis still appears to be clinically useful in the acute evaluation of periprosthetic joint infection in the setting of clinically established infection and a series of negative cultures. Second, we found it easier to obtain synovial fluid samples from patients undergoing revision knee arthroplasty compared with hip arthroplasty, and we therefore combined data from the two joints to increase the power of our analyses. Combination of these two groups, however, may inaccurately conflate different parameters between the two groups. Third, despite the good match between findings from the Ibis and those from conventional culture in settings in which culture identified an organism, there was a single patient in whom we found a discrepancy between the findings from culture (Peptostreptococcus and Corynebacterium) and those from the Ibis (Streptococcus, Enterococcus, and Treponema). Given the high sensitivity of the Ibis, we were surprised to note this discrepancy and can only postulate that some degree of contamination led to this result.

The Ibis appears to be a useful technology for identification of organisms in culture-negative periprosthetic joint infection. Its high sensitivity precludes current use as a diagnostic modality for periprosthetic joint infection, since it appears to also be capable of identifying organisms that are not associated with clinically relevant infection. Nevertheless, we believe these identifications to represent true-positive results, and we believe that these findings hint at the complex pathophysiology of periprosthetic joint infection. Patients whose arthroplasty requires revision for what is considered a noninfectious cause may in fact be experiencing the long-term effects of subclinical infection. We are currently working on expanding our cohort of patients to further analyze the Ibis results and compare them with clinical laboratory findings. We are also seeking to make improvements in our collection and processing techniques. For example, it appears that the stringent methods used for sample collection in the present study (e.g., flash-freezing and transportation on dry ice) are not necessary for analysis by the Ibis; collection methods similar to those used for microbiological cultures are more than adequate. Finally, we believe that it will be extremely useful to expand this project into a multi-institutional endeavor in order to standardize results across different techniques and varying patient populations.

Christina L. Jacovides, BS

Bahar Adeli, BA

Bryan Hozack, BA

Javad Parvizi, MD, FRCS

Rothman Institute of Orthopedics at

Thomas Jefferson University Hospital,

925 Chestnut Street, Philadelphia, PA 19107.

E-mail address for J. Parvizi: research@rothmaninstitute.com

Rachael Kreft, BS, RN

Garth D. Ehrlich, PhD

Center for Genomic Sciences,

Allegheny Singer Research Institute,

320 East North Avenue,

Pittsburgh, PA 15212 
The Journal of Bone \& Joint Surgery $\cdot$ JBjS. org Volume 94-A · Number 24 - December 19, 2012
Identification of Pathogens by Mass Spectrometry in

Culture-Negative Periprosthetic Joint Infection

\section{References}

1. Della Valle C, Parvizi J, Bauer TW, Dicesare PE, Evans RP, Segreti J, Spangehl M, Watters WC 3rd, Keith M, Turkelson CM, Wies JL, Sluka P. Hitchcock K; American Academy of Orthopaedic Surgeons. Diagnosis of periprosthetic joint infections of the hip and knee. J Am Acad Orthop Surg. 2010 Dec;18(12):760-70.

2. Della Valle C, Parvizi J, Bauer TW, DiCesare PE, Evans RP, Segreti J, Spangehl M, Watters WC 3rd, Keith M, Turkelson CM, Wies JL, Sluka P, Hitchcock K; American Academy of Orthopaedic Surgeons. American Academy of Orthopaedic Surgeons clinical practice guideline on: the diagnosis of periprosthetic joint infections of the hip and knee. J Bone Joint Surg Am. 2011 Jul 20;93(14):1355-7.

3. Bauer TW, Parvizi J, Kobayashi N, Krebs V. Diagnosis of periprosthetic infection. J Bone Joint Surg Am. 2006 Apr;88(4):869-82.

4. Parvizi J, Ghanem E, Menashe S, Barrack RL, Bauer TW. Periprosthetic infection: what are the diagnostic challenges? J Bone Joint Surg Am. 2006 Dec;88 Suppl 4:138-47.

5. Trampuz A, Piper KE, Jacobson MJ, Hanssen AD, Unni KK, Osmon DR, Mandrekar JN, Cockerill FR, Steckelberg JM, Greenleaf JF, Patel R. Sonication of removed hip and knee prostheses for diagnosis of infection. N Engl J Med. 2007 Aug 16;357(7):654-63.

6. Zimmerli W, Trampuz A, Ochsner PE. Prosthetic-joint infections. N Engl J Med. 2004 Oct 14;351(16):1645-54.

7. Berbari EF, Marculescu C, Sia I, Lahr BD, Hanssen AD, Steckelberg JM, Gullerud R, Osmon DR. Culture-negative prosthetic joint infection. Clin Infect Dis. 2007 Nov 1;45(9):1113-9. Epub 2007 Sep 26.

8. Lee SC, Jung KA, Yoon JY, Nam CH, Hwang SH, Park IS. Analysis of synovial fluid in culture-negative samples of suspicious periprosthetic infections. Orthopedics. 2010 Oct 11;33(10):725. doi: 10.3928/014774477-20100826-13.

9. Gallo J, Kolar M, Dendis M, Loveckova Y, Sauer P, Zapletalova J, Koukalova D. Culture and PCR analysis of joint fluid in the diagnosis of prosthetic joint infection. New Microbiol. 2008 Jan;31(1):97-104.

10. Gallo J, Raska M, Dendis M, Florschütz AV, Kolár M. Molecular diagnosis of prosthetic joint infection. A review of evidence. Biomed Pap Med Fac Univ Palacky Olomouc Czech Repub. 2004 Dec;148(2):123-9.

11. Kobayashi N, Bauer TW, Sakai H, Togawa D, Lieberman IH, Fujishiro T, Procop GW. The use of newly developed real-time PCR for the rapid identification of bacteria in culturenegative osteomyelitis. Joint Bone Spine. 2006 Dec;73(6):745-7. Epub 2006 Mar 24. 12. Moojen DJ, Spijkers SN, Schot CS, Nijhof MW, Vogely HC, Fleer A, Verbout AJ, Castelein RM, Dhert WJ, Schouls LM. Identification of orthopaedic infections using broad-range polymerase chain reaction and reverse line blot hybridization. J Bone Joint Surg Am. 2007 Jun;89(6):1298-305.

13. Achermann $Y$, Vogt $M$, Leunig $M$, Wüst J, Trampuz A. Improved diagnosis of periprosthetic joint infection by multiplex PCR of sonication fluid from removed implants. J Clin Microbiol. 2010 Apr;48(4):1208-14. Epub 2010 Feb 17.

14. Larson AN, Razonable RR, Hanssen AD. Capnocytophaga canimorsus a novel pathogen for joint arthroplasty. Clin Orthop Relat Res. 2009 Jun;467(6):1634-8. Epub 2008 Dec 9.

15. Bergin PF, Doppelt JD, Hamilton WG, Mirick GE, Jones AE, Sritulanondha S, Helm JM, Tuan RS. Detection of periprosthetic infections with use of ribosomal RNA based polymerase chain reaction. J Bone Joint Surg Am. 2010 Mar;92(3):654-63. 16. Kalogianni DP, Goura S, Aletras AJ, Christopoulos TK, Chanos MG, Christofidou M, Skoutelis A, loannou PC, Panagiotopoulos E. Dry reagent dipstick test combined with 23S rRNA PCR for molecular diagnosis of bacterial infection in arthroplasty. Anal Biochem. 2007 Feb 15;361(2):169-75. Epub 2006 Nov 28.
17. Rasouli MR, Harandi AA, Adeli B, Purtill JJ, Panizi J. Revision total knee arthroplasty: infection should be ruled out in all cases. J Arthroplasty. 2012 Jun;27(6):1239-43.e1-2. Epub 2012 Mar 3

18. Neogi DS, Kumar A, Yadav CS, Singh S. Delayed periprosthetic tuberculosis after total knee replacement: is conservative treatment possible? Acta Orthop Belg. 2009 Feb; 75(1):136-40.

19. Zappe B, Graf S, Ochsner PE, Zimmerli W, Sendi P. Propionibacterium spp. in prosthetic joint infections: a diagnostic challenge. Arch Orthop Trauma Surg. 2008 Oct;128(10):1039-46. Epub 2007 Sep 15.

20. Schäfer P, Fink B, Sandow D, Margull A, Berger I, Frommelt L. Prolonged bacterial culture to identify late periprosthetic joint infection: a promising strategy. Clin Infect Dis. 2008 Dec 1;47(11):1403-9.

21. Sendi P, Frei R, Maurer TB, Trampuz A, Zimmerli W, Graber P. Escherichia coli variants in periprosthetic joint infection: diagnostic challenges with sessile bacteria and sonication. J Clin Microbiol. 2010 May:48(5):1720-5. Epub 2010 Mar 24.

22. Gallo PH, Melton-Kreft R, Nistico L, Sotereanos NG, Sewecke JJ, Stoodley P, Ehrlich GD, Costerton JW, Kathju S. Demonstration of Bacillus cereus in orthopaedicimplant-related infection with use of a multi-primer polymerase chain reaction-mass spectrometric assay: report of two cases. J Bone Joint Surg Am. 2011 Aug 3; 93(15):e85.

23. Stoodley P, Conti SF, DeMeo PJ, Nistico L, Melton-Kreft R, Johnson S, Darabi A, Ehrlich GD, Costerton JW, Kathju S. Characterization of a mixed MRSA/MRSE biofilm in an explanted total ankle arthroplasty. FEMS Immunol Med Microbiol. 2011 Jun;62(1):66-74. doi: 10.1111/j.1574-695X.2011.00793.x. Epub 2011 Mar 22. 24. Kathju S, Lasken RS, Satish L, Johnson S, Stoodley P, Post JC, Ehrlich GD. Multiple displacement amplification as an adjunct to PCR-based detection of Staphylococcus aureus in synovial fluid. BMC Res Notes. 2010 Oct 13;3:259. 25. Costerton W, Veeh R, Shirtliff M, Pasmore M, Post C, Ehrlich G. The application of biofilm science to the study and control of chronic bacterial infections. J Clin Invest. 2003 Nov;112(10):1466-77.

26. Hall-Stoodley L, Costerton JW, Stoodley P. Bacterial biofilms: from the natural environment to infectious diseases. Nat Rev Microbiol. 2004 Feb;2(2):95-108.

27. Gristina AG, Costerton JW. Bacterial adherence to biomaterials and tissue. The significance of its role in clinical sepsis. J Bone Joint Surg Am. 1985 Feb;67(2):264-73. 28. Dobbins JJ, Seligson D, Raff MJ. Bacterial colonization of orthopedic fixation devices in the absence of clinical infection. J Infect Dis. 1988 Jul;158(1):203-5. 29. Nelson CL, McLaren AC, McLaren SG, Johnson JW, Smeltzer MS. Is aseptic loosening truly aseptic? Clin Orthop Relat Res. 2005 Aug;(437):25-30.

30. Parvizi J, Suh DH, Jafari SM, Mullan A, Purtill JJ. Aseptic loosening of total hip arthroplasty: infection always should be ruled out. Clin Orthop Relat Res. 2011 May;469(5):1401-5. Epub 2011 Mar 2.

31. Ince A, Rupp J, Frommelt L, Katzer A, Gille J, Löhr JF. Is "aseptic" loosening of the prosthetic cup after total hip replacement due to nonculturable bacterial pathogens in patients with low-grade infection? Clin Infect Dis. 2004 Dec 1;39(11):1599-603. Epub 2004 Nov 2

32. Günther R, Morawietz L, Gehrke T, Frommelt L, Kaps C, Krenn V. [Inflammatory reactions in the wear particle induced and infectious periprosthetic membrane of loosened hip- and knee endoprostheses: pathogenetic relevance of differentially expressed genes cd9, cd11b, cd18, cd52 and pdgfrbeta]. Orthopade. 2005 Jan;34(1):55-64. German. 